\title{
Irisin Regulates the Functions of Hepatic Stellate Cells
}

\author{
Hanh Nguyen Dong, So Young Park, Cong Thuc Le, Dae-Hee Choi, Eun-Hee Cho \\ Department of Internal Medicine, Kangwon National University School of Medicine, Chuncheon, Korea
}

Background: Hepatic stellate cells (HSCs) are known to play a fundamental role in the progression of liver fibrosis. Once HSCs are activated, they are involved in proliferation, migration, and contractility which are characteristics of liver fibrogenesis. Recent studies have shown that irisin, a myokine secreted during physical exercise, has a protective effect in various metabolic diseases, especially in renal fibrosis. However, whether irisin is involved in HSC activation and other processes associated with liver fibrosis has not yet been investigated. In this study, we reveal the role of irisin in HSC activation as well as in proliferation, migration, and contractile properties of HSCs in vitro.

Methods: LX-2 cells, immortalized human HSCs, were treated with transforming growth factor beta 1 (TGF- $\beta 1$ ), a core regulator of HSC fibrosis, with or without irisin, and markers of the aforementioned processes were analyzed. Further, an inflammatory response was stimulated with TGF- $\beta 1$ and lipopolysaccharide (LPS) in combination with irisin and the expression of cytokines was measured. Results: Recombinant irisin significantly suppressed the expression of TGF- $\beta 1$-stimulated fibrosis markers including alpha-smooth muscle actin and collagen type 1 alpha 1 and prevented the TGF- $\beta 1$-induced proliferation, migration, and contractility of LX-2 cells. Additionally, irisin ameliorated the production of interleukin-6 (IL-6) and IL-1 $\beta$ induced by TGF- $\beta 1$ and LPS treatments.

Conclusion: These findings suggested that irisin potently improved the progression of hepatic fibrosis by regulating HSC activation, proliferation, migration, contractility, and HSC-mediated production of inflammatory cytokine.

Keywords: Hepatic stellate cells; Irisin; Lipopolysaccharides; Liver cirrhosis; Transforming growth factor beta1

\section{INTRODUCTION}

Hepatic stellate cells (HSCs), that account for $5 \%$ to $15 \%$ of the total cells in the normal liver have several physiological functions including storage of vitamin A and production of extracellular matrix (ECM) components [1,2]. During liver injury, HSCs undergo transformation from a quiescent state to an activated state, in which they undergo proliferation, fibrogenesis, and contractility [3,4]. HSCs play a significant role in the development of liver fibrosis, a consequence of wound healing response to various diseases associated with viruses, autoimmuni-

Received: 20 March 2020, Revised: 25 May 2020, Accepted: 17 June 2020

Corresponding author: Eun-Hee Cho

Department of Internal Medicine, Kangwon National University School of

Medicine, 1 Gangwondaehak-gil, Chuncheon 24341, Korea

Tel: +82-33-258-9167, Fax: +82-33-258-2455, E-mail: ehcho@kangwon.ac.kr ty, and drugs [5].

Transforming growth factor beta 1 (TGF- $\beta 1$ ) is a well-known pro-fibrogenic cytokine, which is responsible for HSC transdifferentiation through the SMAD-and non-SMAD-dependent pathway during their initial stages of activation [6]. Further, TGF- $\beta 1$ enhances ECM production and collagen synthesis by HSCs $[7,8]$.

Irisin, a cleaved and secreted fragment from fibronectin type III domain-containing protein 5 (FNDC5), is a myokine released mainly from skeletal muscle into circulation in response to physical exercise [9]. Liver and adipose tissue are other

\section{Copyright $\odot 2020$ Korean Endocrine Society}

This is an Open Access article distributed under the terms of the Creative Commons Attribution Non-Commercial License (https://creativecommons.org/ licenses/by-nc/4.0/) which permits unrestricted non-commercial use, distribution, and reproduction in any medium, provided the original work is properly cited. 
sources of irisin synthesis and secretion on a smaller scale [10]. Initially, irisin was found to enhance browning subcutaneous adipose tissue cells by stimulating uncoupling protein 1 expression, thus its primary role is to increase thermogenesis and energy expenditure [11]. Subsequent studies have shown the positive association of irisin with metabolic diseases including obesity, type 2 diabetes mellitus (T2DM), metabolic (dysfunction) associated fatty liver disease (MAFLD) [12], lipid metabolism, and cardiovascular disease [13]. In an obese mouse model stimulated by high-fat diet, overexpression of FNDC5 remarkably improved glucose/lipid metabolism as well as insulin resistance [14]. In glucose homeostasis, although the relationship between the levels of irisin and the pathogenesis of T2DM remains controversial (which results from the inaccurate methods and reported investigations), irisin was reported to be a potential regulator of glucose metabolism [15].

In damaged kidneys, irisin was found to play the role of a mediator of the process in which overexpression of peroxisome proliferator-activated receptor gamma coactivator 1-alpha subsequently resulted in the suppression of reprogramming and fibrosis [16]. However, to the best of our knowledge, the role of irisin in HSC activation during liver fibrogenesis remains unclear. In this study, we aimed to investigate whether irisin regulated fibrogenic progression in vitro. TGF- $\beta 1$ and lipopolysaccharide (LPS) were used to activate LX-2 cells and the effect of the co-treatment with irisin was evaluated by measuring the expression of protein markers of several important processes related to fibrosis.

\section{METHODS}

\section{Materials}

The reagents used in the study were obtained from the indicated suppliers: TGF- $\beta 1$ from Bio-Techne (Minneapolis, MN, USA); irisin from Cayman Chemical (Ann arbor, MI, USA); LPS from Sigma (St. Louis, MO, USA), antibodies against $\alpha$-smooth muscle actin ( $\alpha$-SMA) from Abcam (Cambridge, England), collagen type 1 alpha 1 (COL1A1) from Sigma-Aldrich (St. Louis, MO, USA), interleukin-6 (IL-6) from Santa Cruz Biotechnology (Santa Cruz, CA, USA), glyceraldehyde 3-phosphate dehydrogenase (GAPDH) from GeneTex (Irvine, CA, USA); cleaved-poly (ADP-ribose) polymerase (PARP), PARP, phosphorylated myosin light chain 2 (p-MLC 2), total myosin light chain 2 (t-MLC 2), and IL-1 $\beta$ from Cell Signaling Technology (Danvers, MA, USA); all other materials were obtained from Sigma.

\section{Cell culture}

LX-2 cells, immortalized human HSCs, were kindly provided by Prof. Ja June Jang of Seoul National University, Korea. The cells were maintained in Dulbecco's modified Eagle's medium (DMEM) containing $100 \mathrm{~mL} / \mathrm{L}$ fetal bovine serum with 100,000 $\mathrm{U} / \mathrm{L}$ penicillin and $100 \mathrm{mg} / \mathrm{L}$ streptomycin and cultured at $37^{\circ} \mathrm{C}$ in a $5 \% \mathrm{CO}_{2}$ incubator.

\section{Proliferation assay}

LX-2 cells were plated $\left(5 \times 10^{4}\right.$ cells/well) in a 24 -well culture dish and incubated at $37^{\circ} \mathrm{C}$ in a $5 \% \mathrm{CO}_{2}$ incubator for 24 hours. Then the media was changed to DMEM without phenol red and TGF- $\beta 1$ or TGF- $\beta 1$ plus irisin was added and the cells were incubated further for 24 hours. A total of $50 \mu \mathrm{L}$ of EZ-Cytox reagent (DoGen, Seoul, Korea) was added to each well and the dishes were incubated for 1 hour. The plate was shaken gently for 1 minute and the absorbance was measured at $450 \mathrm{~nm}$ using a plate reader.

\section{Migration assay}

LX-2 cells were plated $\left(5 \times 10^{5}\right.$ cells/well) in 6-well plates. When the cell reached $90 \%$ confluence, $1 \mathrm{mg} / \mathrm{L}$ mitomycin $\mathrm{C}$ was added and the cells were incubated for 1 hour. After making an injury line with a yellow tip, the cell monolayer was washed with phosphate buffered saline. Then the cells were incubated for 6 hours with TGF- $\beta 1$ or TGF- $\beta 1$ plus irisin in culture medium. Cell migration was observed using microscopy and the changes in the width of the injury lines indicated the distance of migration.

\section{Western blot analyses}

Cells were plated $\left(3 \times 10^{5}\right.$ cells/well $)$ in a 6-well culture dish and incubated at $37^{\circ} \mathrm{C}$ in a $5 \% \mathrm{CO}_{2}$ incubator for 24 hours. Then the medium was replaced with medium containing TGF- $\beta 1$ or TGF- $\beta 1$ plus irisin and cultures were incubated further for 24 hours. Cell lysates were prepared using RIPA buffer (ATTA Corporation, Tokyo, Japan) containing phosphatases and protease inhibitors (ATTA Corporation), followed by quantitation of the proteins with a bicinchoninic acid protein assay kit (Thermo Scientific, Rockford, IL, USA). The mixture of proteins from the cell lysate were separated using sodium dodecyl sulfatepolyacrylamide gel electrophoresis (SDS-PAGE) before being transferred to a polyvinylidene fluoride membrane (Millipore, Bedford, MA, USA). Non-fat dry milk (5\%) in 1 X Tris-buffered saline containing $0.5 \%$ Tween- 20 was used to block the nonspecific binding sites on the membranes for 1 hour at room tem- 
perature, then the membranes were incubated with primary antibodies at $4^{\circ} \mathrm{C}$ overnight or for 48 hours. The membranes were then incubated with the appropriate horseradish peroxidaseconjugated secondary antibodies at room temperature for 80 minutes. Images were taken using a Bio-Rad Chemidoc XRS system (Bio-Rad Laboratories Inc., Hercules, CA, USA) after exposing the membrane to WestGlow PICO PLUS chemiluminescent substrate (Biomax, Seoul, Korea). Image J program version 1.4.3.67 (National Institute of Health [NIH], Bethesda, MD, USA) was used to calculate the intensity of the bands.

\section{Cell contraction assay}

LX-2 cells were cultured and trypsinized to prepare a cell suspension $\left(10^{6}\right.$ cells $\left./ \mathrm{mL}\right)$. The cell suspension was mixed with cold collagen gel working solution (ratio $1: 4$ ) and $0.5 \mathrm{~mL}$ of the mix was added to a 24-well plate. The plate was incubated at $37^{\circ} \mathrm{C}$ for 1 hour for complete collagen polymerization before adding $1 \mathrm{~mL}$ of culture medium over each gel layer. The stressed matrix was developed for 24 hours by keeping the cells in an incubator. The medium was replaced with fresh culture medium with TGF- $\beta 1$ or TGF- $\beta 1$ with irisin and the collagen gels were gently released from the sides of the culture dishes to start the contraction. The diameters of gel contraction were measured using microscopy and used for acreage calculation.

\section{Enzyme-linked immunosorbent assay}

Following the treatment of cells with TGF- $\beta 1$ or TGF- $\beta 1$ plus irisin, the media were collected and kept in the freezer before use. The amounts of IL-6 in were determined in the supernatant in different samples using a KOMA enzyme-linked immunosorbent assay (ELISA) KIT (Komabiotech, Seoul, Korea), following the manufacturer's protocol. Briefly, the plate was coated with the conditioned media for 2 hours, followed by incubation for 2 hours with the detection antibody. The medium was replaced with streptavidin-conjugated horseradish peroxidase and the plate was incubated for 30 minutes. 3,3',5,5'-Tetramethylbenzidine solution was used for an appropriate time for color development before adding the stop solution. The optical density was measured at $450 \mathrm{~nm}$ using a microplate reader. The standard range of human IL-6 according to the ELISA kit was 7.8 to $500 \mathrm{pg} / \mathrm{mL}$.

\section{Statistical analysis}

Data are presented as mean \pm standard error of the mean (SEM) of three or more independent experiments. Differences between the treatment groups were evaluated using the Student's $t$ test with Microsoft Excel version 14.0.7214.5000 (32 bit). Differences were considered significant when $P<0.05$.

\section{RESULTS}

\section{Irisin abolishes hepatic stellate cell activation}

To investigate the role of irisin in HSC activation, LX-2 cells were activated for 24 hours with $6 \mathrm{ng} / \mathrm{mL}$ TGF- $\beta 1$ and co-treated with $100 \mathrm{nM}$ irisin. The protein level of fibrogenesis markers like $\alpha$-SMA, COL1A1, and the housekeeping gene GAPDH were measured by Western blotting. TGF- $\beta 1$ treatment markedly increased the protein level of $\alpha$-SMA, COL1A1, and co-treatment with irisin significantly reduced these levels (Fig. 1A).

\section{Irisin attenuates migration of activated HSCs}

Migration is a fundamental feature of activated HSCs and is influenced by TGF- $\beta 1$ through various pathways [17]. To clarify whether irisin affects this process, we treated LX-2 cells with TGF- $\beta 1$ or combination of TGF- $\beta 1$ and irisin, followed by wound healing assay. Migration of the TGF- $\beta 1$-activated LX-2 cells decreased after co-culturing for 6 hours with $6 \mathrm{ng} / \mathrm{mL}$ of TGF- $\beta 1$ and irisin (100 nM) (Fig. 1B).

\section{Irisin reduces contractility of activated HSCs}

We investigated whether irisin affects contractile property using gel contractility assay and confirmed the effect using Western blotting. In the gel contractility assay, TGF- $\beta 1$ significantly enhanced the contraction rate of LX-2 cells after 24 hours of treatment, which was reversed by irisin (Fig. 2A). In Western blotting, the ratio of the active and total forms of the contractile regulatory protein, myosin light chain 2 , was found to be lower in the TGF- $\beta 1$ and irisin co-treatment group in comparison with the only TGF- $\beta 1$ treatment group (Fig. 2B).

\section{Irisin reduces proliferation but is not involved in cell apoptosis of activated HSCs}

As shown in Fig. 3A, TGF- $\beta 1$ enhanced the proliferation of LX-2 cells after 24 hours of treatment which was attenuated by irisin. To identify the underlying mechanism, we assessed the level of the apoptotic markers levels using Western blotting. We found that TGF- $\beta 1$ maintained the survival of LX- 2 cells evidenced by a reduction in the ratio of cleaved-PARP and PARP compared to the control group. However, there was no statistically significant difference between TGF- $\beta 1$ and TGF- $\beta 1$ plus irisin groups, which suggested that irisin was not involved in this pathway (Fig. 3B). 

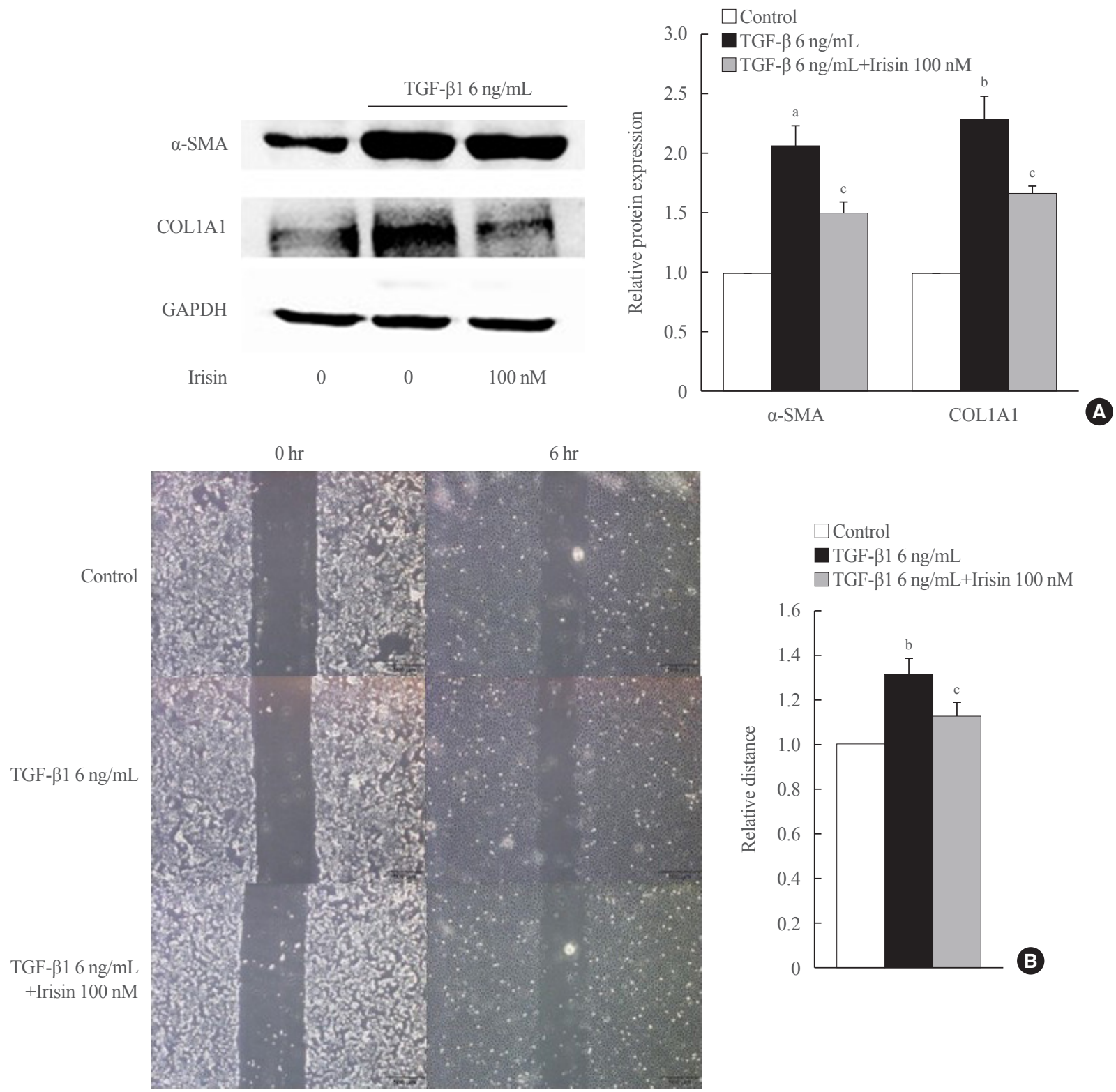

Fig. 1. Irisin abolishes hepatic stellate cell activation and migration. (A) LX-2 cells were stimulated with transforming growth factor beta 1 (TGF- $\beta 1 ; 6 \mathrm{ng} / \mathrm{mL})$ or TGF- $\beta 1$ plus irisin $(100 \mathrm{nM})$ for 24 hours. Western blotting was conducted to measure the level of fibrogenesis proteins, alpha-smooth muscle actin ( $\alpha$-SMA) and collagen type 1 alpha 1 (COL1A1). Band intensities were measured using ImageJ software (National Institutes of Health) and are plotted to the right of the membrane images. Protein levels were normalized to glyceraldehyde 3-phosphate dehydrogenase (GAPDH). (B) Wound healing migration assay was used to investigate the effect of irisin (100 nM) on the migration of LX-2 cells following 6 hours of co-treatment with TGF- $\beta 1(6 \mathrm{ng} / \mathrm{mL})$. ${ }^{\mathrm{a}} P<0.001$; ${ }^{\mathrm{b}} P<0.01 \mathrm{vs}$. control; ${ }^{\mathrm{c}} P<0.05 \mathrm{vs}$. TGF- $\beta 1$ only treatment (mean \pm standard error of the mean, $n=3$ ).

\section{Irisin attenuates TGF- $\beta 1$-induced inflammatory cytokine expression in HSCs}

To determine the role of TGF- $\beta 1$ and irisin in the production and secretion of inflammatory cytokines (IL-6 and IL-1 $\beta$ ), Western blot analysis and ELISA were performed using cell lysate and culture medium, respectively following stimulation of the LX-2 cells with $6 \mathrm{ng} / \mathrm{mL}$ TGF- $\beta 1$ with or without $100 \mathrm{nM}$ irisin for 24 hours. Western blot analysis of the cell lysates revealed that iri$\sin$ lowered the expression of IL- 6 and IL- $1 \beta$ in the TGF- $\beta 1$ stimulated cells. ELISA revealed that the addition of irisin to LX-2 cells suppressed of secretion of IL-6 into the culture medium (Fig. 4A). 

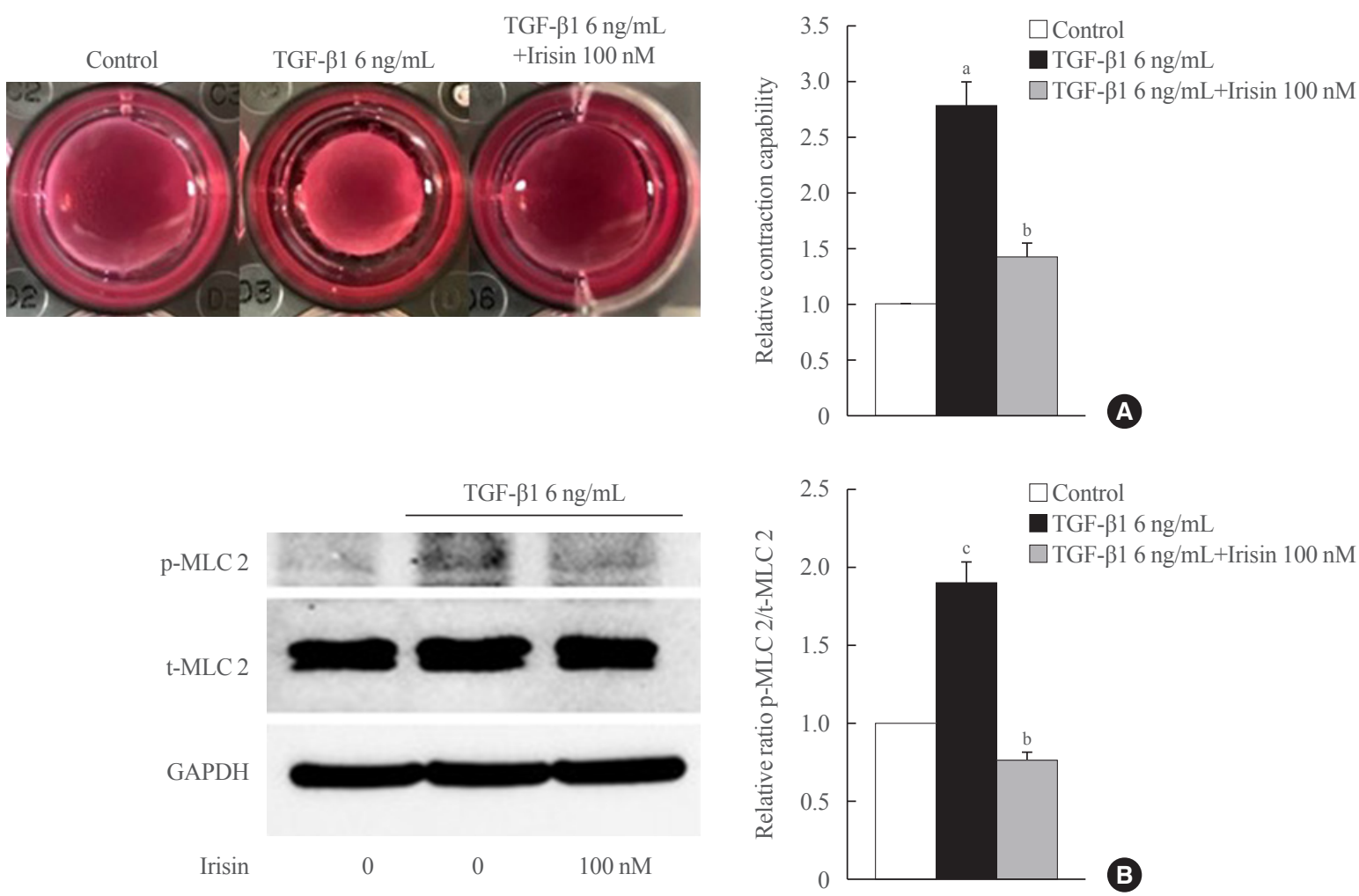

Fig. 2. Irisin reduces the contractility of activated hepatic stellate cells. LX-2 cells were treated with transforming growth factor beta 1 (TGF- $\beta 1 ; 6 \mathrm{ng} / \mathrm{mL}$ ) or TGF- $\beta 1$ plus irisin $(100 \mathrm{nM})$ for 24 hours. (A) Cell contraction assay was used to test contraction capability, (B) Western blotting was used to analyze the ratio of the different forms of the contractile marker, phosphorylated myosin light chain 2 (p-MLC 2) and total myosin light chain 2 (t-MLC 2) in LX-2 lysates. Band intensities were measured using ImageJ software (National Institutes of Health) and are plotted to the right of the membrane images. Protein levels were normalized with glyceraldehyde 3-phosphate dehydrogenase (GAPDH). ${ }^{\mathrm{a}} P<0.01$ vs. control; ${ }^{\mathrm{b}} P<0.01 \mathrm{vs}$. TGF- $\beta 1$ only treatment (mean \pm standard error of the mean, $n=3$ ); ${ }^{\mathrm{c}} P<0.05$ vs. control.
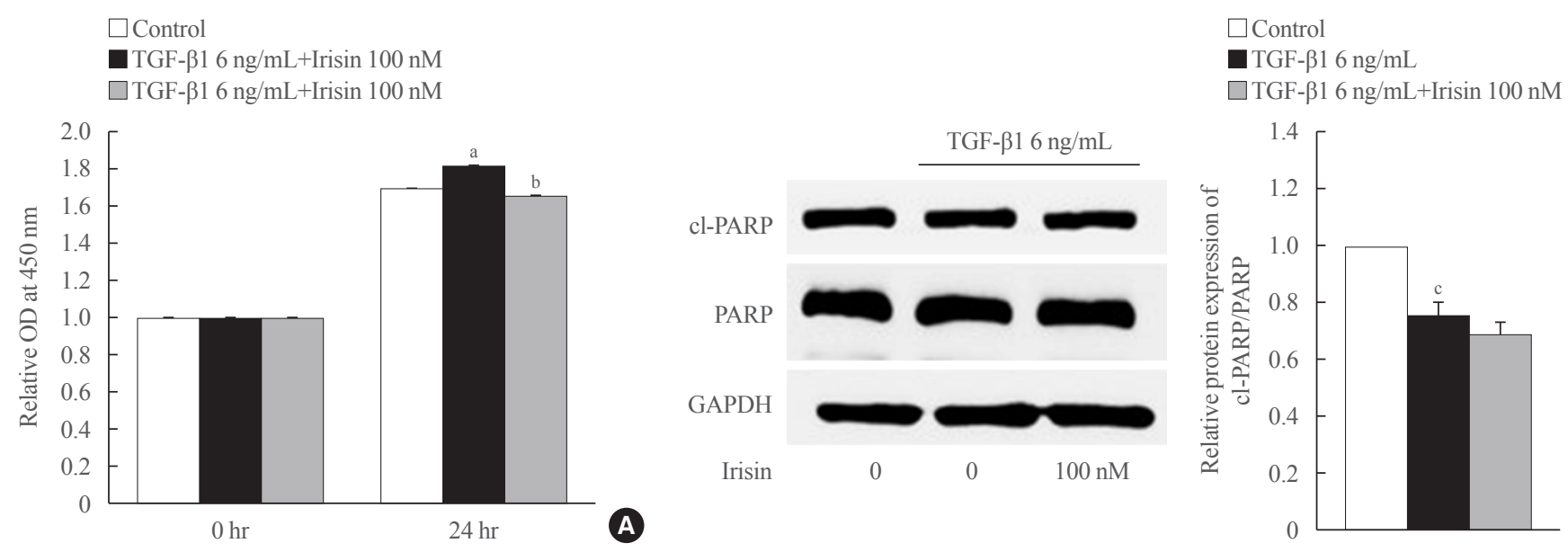

Fig. 3. Irisin inhibits proliferation of activated hepatic stellate cell but is not involved in the apoptotic pathway. LX-2 cells were treated with transforming growth factor beta 1 (TGF- $\beta 1 ; 6 \mathrm{ng} / \mathrm{mL}$ ) or TGF- $\beta 1$ plus irisin (100 nM) for 24 hours. (A) Cell proliferation was measured using EZ-Cytox assay kit (DoGen). The optical density (OD) was measured at $450 \mathrm{~nm}$. (B) Western blotting was used to analyze the apoptotic markers, and poly (ADP-ribose) polymerase (PARP) and cleaved-PARP (cl-PARP). Band intensities were measured using ImageJ software (National Institutes of Health) and are plotted to the right of the membrane images. Protein levels were normalized to glyceraldehyde 3 -phosphate dehydrogenase (GAPDH). ${ }^{\mathrm{a}} P<0.01$ vs. control; ${ }^{\mathrm{b}} P<0.01 \mathrm{vs.}$ TGF- $\beta 1$ only treatment (mean \pm standard error of the mean [SEM], $n=6)$; ${ }^{\mathrm{c}} P<0.001$ vs. control (mean \pm SEM, $n=7$ ). 

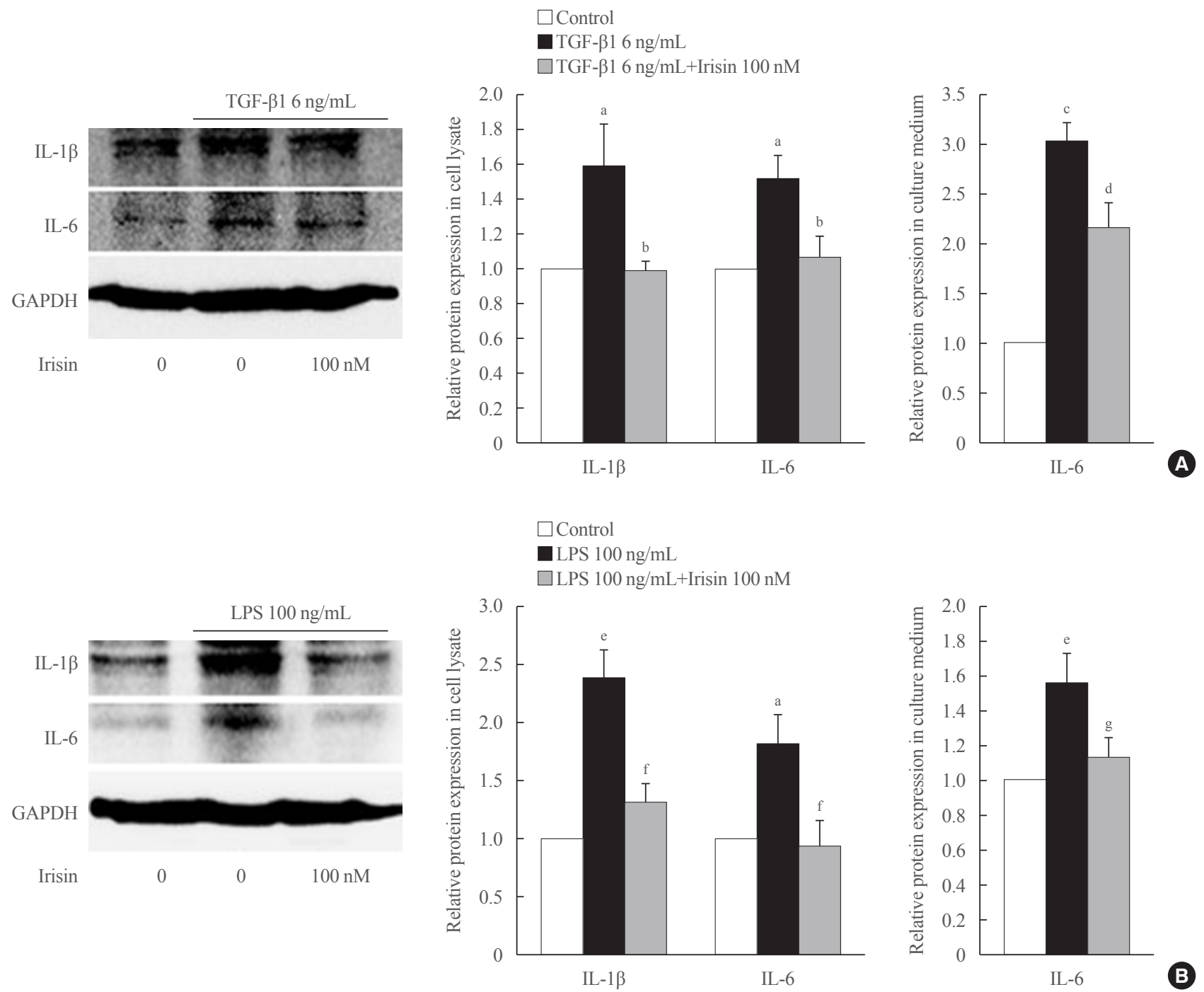

Fig. 4. Irisin attenuates transforming growth factor beta 1 (TGF- $\beta 1$ )- and lipopolysaccharide (LPS)-induced inflammatory cytokine expression in hepatic stellate cells. LX-2 cells were treated with (A) TGF- $\beta 1(6 \mathrm{ng} / \mathrm{mL})$ or (B) LPS (100 ng/mL) with recombinant of irisin (100 $\mathrm{nM}$ ) for 24 hours. Western blotting was used to detect the expression of inflammatory cytokines, interleukin 6 (IL-6) and IL-1 $\beta$ in the cell lysates. Band intensities were measured using ImageJ software (National Institutes of Health) and are plotted to the right of the membrane images. Protein levels were normalized to glyceraldehyde 3-phosphate dehydrogenase (GAPDH). Enzyme-linked immunosorbent assay (ELISA) was used to measure secretion of IL- 6 in the cell culture medium. ${ }^{\mathrm{a}} P<0.05$ vs. control; ${ }^{\mathrm{b}} P<0.05$ vs. TGF- $\beta 1$ only treatment (mean \pm standard error of the mean [SEM], $n=7$ for IL-6 and $n=5$ for IL-1 $\beta$ ); ${ }^{\mathrm{c}} P<0.001$ vs. control; ${ }^{\mathrm{d}} P<0.05$ vs. TGF- $\beta 1$ only treatment (mean \pm SEM, $n=3$ ); ${ }^{\mathrm{e}} P<0.01$ vs. control; ${ }^{\mathrm{f}} P<0.05$ vs. LPS only treatment (mean \pm SEM, $n=3$ for IL- $1 \beta, n=4$ for IL-6); ${ }^{\mathrm{g}} P<0.05$ vs. LPS only treatment (mean \pm SEM, $n=5)$.

\section{Irisin ameliorates LPS-induced hepatic stellate cell activation and inflammatory cytokine expression}

When stimulated with LPS for 24 hours, the expression of $\alpha$-SMA and COL1A1 increases in LX-2 and the addition of 100 $\mathrm{nM}$ irisin reduces the protein expression (data not shown). As shown in Fig. 4B, treatment with recombinant irisin reduced the LPS-induced expression of both IL- 6 and IL- $1 \beta$ in the cell ly- sates. This trend in the change in IL-6 level in culture medium following irisin treatment is confirmed using ELISA.

\section{DISCUSSION}

During hepatic injury, the activation of HSCs results in the disruption of the liver cytoarchitecture finally causing cirrhosis and 
liver failure [18]. Therefore, targeting the HSC-mediated responses in the injured liver are potential anti-fibrotic therapeutic strategies [19].

The results in this study confirmed the effect of TGF- $\beta 1$ (at the concentration of $6 \mathrm{ng} / \mathrm{mL}$ ) on the trans-differentiation of HSCs. During TGF- $\beta 1$ treatment for 24 hours, LX- 2 cells undergo activation, which is evidenced by higher expression of $\alpha$-SMA and COL1A1 proteins. An increase in the contractile proteins such as $\alpha$-SMA in early stages of fibrosis leads to cellular contractility, and TGF- $\beta 1$ was shown to be involved in this pathway using both gel contractility assay and Western blot analysis. Furthermore, the role of TGF- $\beta 1$ as a master regulator in HSC activation was shown through promotion of LX-2 cell migration and boosting of LX-2 cell proliferation.

Our results also showed the protective effect of irisin in HCS activation and the associated processes. The addition of irisin significantly reverses the transformation of HSCs from quiescent HSCs to $\alpha$-SMA expressing contractile myofibroblast evidenced by reduced level of $\alpha$-SMA and suppression of contractility. Through these effects, irisin may positively influence vascular distortion and vascular resistance; thereby reduce portal hypertension which develops during liver injury. Irisin could ameliorate ECM production by HSCs by decreasing the expression of TGF- $\beta 1$-stimulated COL1A1, which in turn slows down the growth of a scar at the site of injury. The accumulation of HSCs through proliferation and migration, during activation with TGF- $\beta 1$, was also significantly inhibited by irisin. From these results, it is obvious that irisin can reverse HCS activation; thereby constituting a promising component of fibrosis regression.

Inflammation also fundamentally participates in the activation of resting HSCs, and in turn exacerbates the trans-differentiation of HSCs into myofibroblasts [20]. Also, activated HSCs themselves can produce a wide range of pro-inflammatory cytokines and chemokines that may trigger the initiation of fibrogenesis $[21,22]$. In our studies, recombinant irisin inhibited TGF- $\beta 1$-induced IL-6 secretion in the cell lysate and cell culture medium of LX-2 cells. According to Robert et al. [23], IL-1 $\beta$ was undetectable in the cell culture medium, and therefore we assessed the level of this cytokine in the cell lysate and expression trend was similar to that of IL-6. These results enabled the identification of an anti-inflammatory role of irisin in HSCs.

LPS, a component in the cell-wall of gram-negative bacteria, is widely known as one of the strongest inflammatory simulators which targets various types of cells including Kupffer cells and HSCs in liver injury $[24,25]$. The anti-inflammatory effect of irisin was reported in LPS-activated macrophages, where it decreases the level of inflammatory cytokines in the Toll-like receptor 4/myeloid differentiation primary response 88-dependent pathways [26]. Similar to these results, when LPS was used as an inflammatory stimulator in our experiments, cotreatment with irisin reduced the level of IL- 6 and IL- $1 \beta$ in the cell lysates and reduced IL-6 secretion into the medium. Irisin was shown to clearly reverse the effect of LPS in the activation and inflammatory response of HSCs.

Despite the inconsistency in reports concerning the concentration of irisin in human serum or plasma (between 0.01 and $2,000 \mathrm{ng} / \mathrm{mL}$ ), a number of studies have investigated the role of irisin in metabolic diseases [27-29]. Irisin aids T2DM by enhancing the conversion of white adipose tissue to brown adipose tissue, improving glucose uptake to the cell in heart and skeletal muscles, increasing the metabolism of glucose and lipid, and the function of pancreatic $\beta$ cells [30-32]. Furthermore, irisin may have a positive effect on inflammatory and de novo lipogenesis, which are contributing factors to the MAFLD pathogenesis. A few studies have reported a negative correlation between the expression of FNDC5, the precursor of irisin, and tumor necrosis factor- $\alpha(\mathrm{TNF}-\alpha)$, and a positive correlation with anti-inflammatory factor, IL-10 [33]. The anti-inflammatory effect of irisin in palmitic acid-treated hepatocytes was evidenced by the reduction in inflammatory cytokine and other inflammatory mediators like TNF- $\alpha$ and IL-6. In another study, recombinant irisin was shown to reduce palmitic acid-induced lipogenesis in AML12 cells and primary hepatocytes [34].

The biggest limitation of our study was using only in vitro experiment to prove the role of irisin in liver fibrosis. In addition to our study, some papers used animal models in their experiments and gained some promising results. In the mouse model, it was reported that HFD-induced liver fibrosis was exacerbated by FNDC5 deficiency and AMPK is molecular which is participated in the inhibition of HSCs activation by FNDC5 [35]. Similarly, irisin was proved to play a protective role against steatosis in mouse hepatocytes which are treated by palmitic acid [31]. According to Peng et al. [16], administration of irisin $(200 \mu \mathrm{g} / \mathrm{kg} /$ day, intraperitoneal injection) for 4 weeks in mice-treated previously by folic acid improved kidney function and renal fibrosis, signified by lowering serum creatinine levels and suppressed expression of the fibrotic protein, respectively.

On the other hand, recent study showed that serum irisin levels and hepatic irisin mRNA levels are positively associated with liver damage in humans and irisin in concentration of 500 $\mathrm{ng} / \mathrm{mL}$ ( 15.6 to $41.7 \mathrm{nM}$ ) induced increased expression of fibrogenesis in activated HSCs [36] suggesting the question of irisin 
as a protective myokine. This study showed pro-fibrogenic role of irisin in hepatic fibrosis contrary to our protective role of irisin in HSCs activation. We did not identify the reason of opposite action of irisin but we suggested that irisin might be profibrogenic in low concentration [36] but anti-fibrogenic in high concentration such as $100 \mathrm{nM}$. Regarding the positive association of serum irisin levels and liver disease severity, the increased irisin levels might be a compensatory response to liver damage.

In the current study, we determined a protective role of irisin based on in vitro experiments. Further studies are needed to understand the influence of irisin on a therapeutic scale. Moreover, the molecular mechanism by which irisin attenuates TGF- $\beta 1$ induced HSC activation and other responses related to proliferation, migration, contractility, and inflammatory remain to be clarified. Further investigations are needed to understand the mechanism through which irisin affects different pathways both in vitro and in vivo studies and to shed light on the regulatory role of irisin in HSCs. A large clinical studies are necessary to fully clarify the clinical association between serum irisin concentrations and various liver disease.

In conclusion, we found that irisin, a myokine produced by skeletal muscles during exercise, can be a potential solution for liver fibrosis by regulating HSC activation and inhibiting other associated responses, including proliferation, contractility, migration and inflammation.

\section{CONFLICTS OF INTEREST}

No potential conflict of interest relevant to this article was reported.

\section{ACKNOWLEDGMENTS}

This research was supported by NRF-2019R1I1A3A01058672 and 2017 Research Grant from Kangwon National University (No. 520170423).

\section{AUTHOR CONTRIBUTIONS}

Conception or design: H.N.D., D.H.C., E.H.C. Acquisition, analysis, or interpretation of data: H.N.D., S.Y.P., C.T.L., E.H.C. Drafting the work or revising: H.N.D., E.H.C. Final approval of the manuscript: D.H.C., E.H.C.

\section{ORCID}

Hanh Nguyen Dong https://orcid.org/0000-0001-6248-7510

Eun-Hee Cho https://orcid.org/0000-0002-1349-8894

\section{REFERENCES}

1. Li J, Zhao YR, Tian Z. Roles of hepatic stellate cells in acute liver failure: from the perspective of inflammation and fibrosis. World J Hepatol 2019;11:412-20.

2. Yin C, Evason KJ, Asahina K, Stainier DY. Hepatic stellate cells in liver development, regeneration, and cancer. J Clin Invest 2013;123:1902-10.

3. Friedman SL. Hepatic stellate cells: protean, multifunctional, and enigmatic cells of the liver. Physiol Rev 2008;88:12572.

4. Lee UE, Friedman SL. Mechanisms of hepatic fibrogenesis. Best Pract Res Clin Gastroenterol 2011;25:195-206.

5. Aydin MM, Akcali KC. Liver fibrosis. Turk J Gastroenterol 2018;29:14-21.

6. Dewidar B, Meyer C, Dooley S, Meindl-Beinker AN. TGF- $\beta$ in hepatic stellate cell activation and liver fibrogenesis: updated 2019. Cells 2019;8:1419.

7. Dewidar B, Soukupova J, Fabregat I, Dooley S. TGF- $\beta$ in hepatic stellate cell activation and liver fibrogenesis: updated. Curr Pathobiol Rep 2015;3:291-305.

8. Fabregat I, Caballero-Diaz D. Transforming growth factor$\beta$-induced cell plasticity in liver fibrosis and hepatocarcinogenesis. Front Oncol 2018;8:357.

9. Huh JY, Panagiotou G, Mougios V, Brinkoetter M, Vamvini MT, Schneider BE, et al. FNDC5 and irisin in humans: I. Predictors of circulating concentrations in serum and plasma and II. mRNA expression and circulating concentrations in response to weight loss and exercise. Metabolism 2012;61: 1725-38.

10. Roca-Rivada A, Castelao C, Senin LL, Landrove MO, Baltar J, Belen Crujeiras A, et al. FNDC5/irisin is not only a myokine but also an adipokine. PLoS One 2013;8:e60563.

11. Bostrom P, Wu J, Jedrychowski MP, Korde A, Ye L, Lo JC, et al. A PGC1- $\alpha$-dependent myokine that drives brown-fatlike development of white fat and thermogenesis. Nature 2012;481:463-8.

12. Eslam M, Sanyal AJ, George J; International Consensus Panel. MAFLD: a consensus-driven proposed nomenclature for metabolic associated fatty liver disease. Gastroenterology 2020;158:1999-2014. 
13. Polyzos SA, Anastasilakis AD, Efstathiadou ZA, Makras P, Perakakis N, Kountouras J, et al. Irisin in metabolic diseases. Endocrine 2018;59:260-74.

14. Xiong XQ, Chen D, Sun HJ, Ding L, Wang JJ, Chen Q, et al. FNDC5 overexpression and irisin ameliorate glucose/lipid metabolic derangements and enhance lipolysis in obesity. Biochim Biophys Acta 2015;1852:1867-75.

15. Perakakis N, Triantafyllou GA, Fernandez-Real JM, Huh JY, Park KH, Seufert J, et al. Physiology and role of irisin in glucose homeostasis. Nat Rev Endocrinol 2017;13:324-37.

16. Peng H, Wang Q, Lou T, Qin J, Jung S, Shetty V, et al. Myokine mediated muscle-kidney crosstalk suppresses metabolic reprogramming and fibrosis in damaged kidneys. Nat Commun 2017;8:1493.

17. Fabregat I, Moreno-Caceres J, Sanchez A, Dooley S, Dewidar B, Giannelli G, et al. TGF- $\beta$ signalling and liver disease. FEBS J 2016;283:2219-32.

18. Bataller R, Brenner DA. Liver fibrosis. J Clin Invest 2005; 115:209-18.

19. Ezhilarasan D, Sokal E, Najimi M. Hepatic fibrosis: it is time to go with hepatic stellate cell-specific therapeutic targets. Hepatobiliary Pancreat Dis Int 2018;17:192-7.

20. Tacke F, Weiskirchen R. Update on hepatic stellate cells: pathogenic role in liver fibrosis and novel isolation techniques. Expert Rev Gastroenterol Hepatol 2012;6:67-80.

21. De Minicis S, Seki E, Uchinami H, Kluwe J, Zhang Y, Brenner DA, et al. Gene expression profiles during hepatic stellate cell activation in culture and in vivo. Gastroenterology 2007;132:1937-46.

22. Seki E, De Minicis S, Osterreicher CH, Kluwe J, Osawa Y, Brenner DA, et al. TLR4 enhances TGF-beta signaling and hepatic fibrosis. Nat Med 2007;13:1324-32.

23. Robert S, Gicquel T, Bodin A, Lagente V, Boichot E. Characterization of the MMP/TIMP imbalance and collagen production induced by IL- $1 \beta$ or TNF- $\alpha$ release from human hepatic stellate cells. PLoS One 2016;11:e0153118.

24. Harvey SA, Dangi A, Tandon A, Gandhi CR. The transcriptomic response of rat hepatic stellate cells to endotoxin: implications for hepatic inflammation and immune regulation. PLoS One 2013;8:e82159.

25. Seki E, Tsutsui H, Nakano H, Tsuji N, Hoshino K, Adachi O, et al. Lipopolysaccharide-induced IL-18 secretion from murine Kupffer cells independently of myeloid differentiation factor 88 that is critically involved in induction of production of IL-12 and IL-1beta. J Immunol 2001;166:2651-7.

26. Mazur-Bialy AI, Pochec E, Zarawski M. Anti-inflammatory properties of irisin, mediator of physical activity, are connected with TLR4/MyD88 signaling pathway activation. Int J Mol Sci 2017;18:701.

27. Crujeiras AB, Pardo M, Casanueva FF. Irisin: 'fat' or artefact. Clin Endocrinol (Oxf) 2015;82:467-74.

28. Polyzos SA, Mathew H, Mantzoros CS. Irisin: a true, circulating hormone. Metabolism 2015;64:1611-8.

29. Polyzos SA, Mantzoros CS. An update on the validity of irisin assays and the link between irisin and hepatic metabolism. Metabolism 2015;64:937-42.

30. Liu S, Du F, Li X, Wang M, Duan R, Zhang J, et al. Effects and underlying mechanisms of irisin on the proliferation and apoptosis of pancreatic $\beta$ cells. PLoS One 2017;12:e175498.

31. Park MJ, Kim DI, Choi JH, Heo YR, Park SH. New role of irisin in hepatocytes: the protective effect of hepatic steatosis in vitro. Cell Signal 2015;27:1831-9.

32. Zhang Y, Li R, Meng Y, Li S, Donelan W, Zhao Y, et al. Irisin stimulates browning of white adipocytes through mitogen-activated protein kinase p38 MAP kinase and ERK MAP kinase signaling. Diabetes 2014;63:514-25.

33. Moreno-Navarrete JM, Ortega F, Serrano M, Guerra E, Pardo $\mathrm{G}$, Tinahones F, et al. Irisin is expressed and produced by human muscle and adipose tissue in association with obesity and insulin resistance. J Clin Endocrinol Metab 2013;98:E769-78.

34. Tang H, Yu R, Liu S, Huwatibieke B, Li Z, Zhang W. Irisin inhibits hepatic cholesterol synthesis via AMPK-SREBP2 signaling. EBioMedicine 2016;6:139-48.

35. Zhou B, Ling L, Zhang F, Liu TY, Zhou H, Qi XH, et al. Fibronectin type III domain-containing 5 attenuates liver fibrosis via inhibition of hepatic stellate cell activation. Cell Physiol Biochem 2018;48:227-36.

36. Petta S, Valenti L, Svegliati-Baroni G, Ruscica M, Pipitone RM, Dongiovanni P, et al. Fibronectin type III domain-containing protein 5 rs3480 A $>$ G polymorphism, irisin, and liver fibrosis in patients with nonalcoholic fatty liver disease. J Clin Endocrinol Metab 2017;102:2660-9. 